\title{
A Serial Reaction Time (SRT) task with symmetrical joystick responding for nonhuman primates
}

\author{
Lisa A. Heimbauer - Christopher M. Conway • \\ Morten H. Christiansen • Michael J. Beran • \\ Michael J. Owren
}

Published online: 18 December 2011

(C) Psychonomic Society, Inc. 2011

\begin{abstract}
The serial reaction time (SRT) task is a simple procedure in which participants produce differentiated responses to each of a series of stimuli presented at varying locations. Learning about stimulus order is revealed through decreased latencies for structured versus randomized sequences. Although widely used with humans and well suited to nonhumans, this paradigm is little used in comparative research. In the present article, we describe an SRT procedure that uses colored circles as stimuli, a circular layout of locations, and symmetrical joystick deflections as responses. In two experiments, we showed that four rhesus macaques (Macaca mulatta) learned to track sequences up to eight items long, with three animals showing faster responding to repeating sequences than to randomized versions. After extended training, these participants also showed evidence of faster responding at all positions within repeating sequences. This method minimizes response effort, equates effort and travel distance across stimulus locations, and is applicable to any joystick-capable species.
\end{abstract}

Keywords Serial reaction time task · Sequence learning . Visual stimuli $\cdot$ Symmetrical joystick responding

\footnotetext{
L. A. Heimbauer $(\bowtie) \cdot$ M. J. Beran · M. J. Owren

Department of Psychology, Georgia State University, P.O. Box 5010, Atlanta, GA 30302-5010, USA

e-mail: lisa.heimbauer@gmail.com

C. M. Conway

Saint Louis University,

St. Louis, MO, USA

M. H. Christiansen

Cornell University,

Ithaca, NY, USA
}

The serial reaction time (SRT) task, as presented by Nissen and Bullemer (1987), is a paradigm in which a participant responds to sequences of stimuli that appear one-by-one at various locations on a computer screen. In their basic procedure, for example, university students tracked a series of asterisks shown on a monitor by quickly pushing a corresponding key located below each stimulus. The SRT task has proven to be easy to implement with either humans or nonhumans and is remarkably versatile. In the present article, we describe a variant of the procedure based on tracking visually presented sequences on a monitor and deflecting a joystick. Stimuli and corresponding responses are arranged in a symmetrical fashion, allowing standardized, easy, and rapid responses. The procedure is illustrated using data from four rhesus monkeys (Macaca mulatta) that were tested with both repeating and random sequences of up to eight items occurring in four different display locations.

\section{SRT testing in humans and nonhumans}

The SRT rationale is illustrated by Nissen and Bullemer's (1987) key-pressing paradigm, in which typical and cognitively impaired participants learned 10 -item sequences involving four stimulus locations. To show learning, the experimenters compared mean reaction times (RTs) for participants responding to stimuli appearing in a repeating sequence of locations to a second group that experienced randomized location orders. Over blocks of trials, individuals tested with repeating sequences showed decreased RTs relative to those experiencing randomized sequences. Variants of this basic procedure have since become widely used with human participants - for instance, in testing sensitivity to probabilistically structured sequencing (e.g., Cleeremans 
\& McClelland, 1991; Hunt \& Aslin, 2001), implicit versus explicit memory (e.g., Willingham \& Goedert-Eschmann 1999), cognitive development (e.g., Thomas \& Nelson, 2001), perceptual- versus motor-based learning (Willingham, 1999), and neuropsychological impairment (e.g., Gabrieli, 1998; Robertson, 2007).

The SRT task is also well suited to testing nonhumans. For example, Christie and colleagues (Christie \& Dalrymple-Alford, 2004; Christie \& Hersch, 2004) developed a version for rodents, with the animals "nose-poking" illuminated apertures in the test chamber wall. In this approach, subjects were first trained on a four-item, repeating sequence of nose-poke locations, and were then switched to randomized sequences to look for "interference effects" indicated by slower latency outcomes (see also Domenger \& Schwarting, 2005, 2007). Birds have been tested using SRT procedures as well, including pigeons studied by Froehlich, Herbranson, Loper, Wood, and Shimp (2004). These investigators used a test chamber with three response keys and showed learning effects for repeating, nine-item sequences based on these horizontally arrayed locations. Latencies were compared for eight different repeating sequences versus randomized versions tested in separate sessions. Overall, the pigeons were found to resemble humans in showing sensitivity to both global and local statistical properties of the sequences.

SRT studies with nonhuman primates have primarily relied on touchscreen displays, with subjects reaching toward each stimulus item as it appears. For example, Procyk, Dominey, Amiez, and Joseph (2000) tested two rhesus monkeys with an illuminated square that could appear in three or four of nine possible locations with repeating versus randomized sequencing. Lee (2003; Lee \& Quessy, 2003) tested two rhesus monkeys in a touchscreen-based paradigm, looking for learning-related changes in single-neuron responses. Their animals experienced 10-item sequences that could include up to five different locations. Locurto and colleagues (Locurto, Gagne, \& Levesque, 2009; Locurto, Gagne, \& Nutile, 2010) extended the touchscreen procedure to cottontop tamarins (Saguinus oedipus), testing three individuals on repeating versus randomized sequences that could include five locations. Finally, Turner, McCairn, Simmons, and Bar-Gad's (2005) version involved rhesus monkeys using a joystick and cursor to make "touching" each item a virtual event. This change was a natural extension of previous work, since laboratory primates have been using joysticks successfully for at least 50 years (e.g., King, 1961; reviewed by Washburn, Beran, Evans, Hoffman, \& Flemming, in press). In Turner et al.'s procedure, the monkeys completed four-item sequences, responding to a dot appearing in a corner of the screen. The response was to move a cursor from the center to that corner and back. The researchers used repeating and randomized sequences to demonstrate that motor learning was occurring, and looked for corresponding changes in basal ganglia activity.

\section{A symmetrical, joystick-based SRT paradigm}

The SRT method described presently builds on the paradigms previously used with rodents, birds, and primates, but is designed for a long-term goal of testing primates for sensitivity to rule-based, grammar-like structure. Achieving that goal was deemed to require testing with a large number of different, but related, sequences, in turn requiring a quick and easy response behavior suitable for long test sessions and extensive practice. The best response would be as standardized as possible, specifically avoiding any systematic latency effects created by moving or reaching to different physical locations. Nose poking, key pecking, and screen touching are simple and intuitive behaviors for the species performing them, but necessarily confound latency and item-specific differences in motor movement and travel time as subjects respond. Locurto et al. (2009) at least partly offset such effects by matching the cumulative travel distances of experimental and control sequences. However, that approach makes it difficult to test a large number of different sequences, and also restricts the degree of randomization on comparison trials.

Two additional considerations were to maximize sensitivity to potential learning effects and to be able to test for these effects both globally, over entire sequences, and locally, between individual items within those sequences. Maximizing sensitivity was addressed through the considerations mentioned earlier, namely aiming for a quick, easy, and standardized response. Furthermore, global learning effects can be examined in any SRT procedure through testing for overall interference effects evidenced by performance differences between structured and randomized sequences. Testing for local effects is more challenging, however, first of all requiring a suitably large number of trials and sessions (cf. Christie \& Dalrymple-Alford, 2004). Statistical dependencies then can be sought in latencies for adjacent or otherwise related items in the sequences (e.g., Froehlich et al., 2004).

The approach used with monkeys by Locurto and colleagues (Locurto et al., 2009, 2010) was to introduce a new element to the classic SRT design, namely including distinct graphical icons that were associated with each SRT location. The tamarins could then be shown to "appreciate" (Locurto et al., 2009) an item's relative position in the list through preference testing with pair-wise presentations of positionand location-specific icons. The animals were, for example, shown to generally prefer icons associated with later sequence positions that were closer to reinforcement, while also being sensitive to the relative positions of icons that occurred earlier and not in close proximity to reinforcement (Locurto et al., 2010). 
This approach was not deemed suitable for our purposes, because introducing icons associated with particular locations and sequence positions represents a significant departure from methods used to test grammar-based sequence learning in humans (e.g., Jamieson \& Mewhort, 2005). More importantly, our priority was to demonstrate that subjects learned-or alternatively did not learn - rule-based probabilistic relationships between all adjacent items in a sequence of up to eight items long, with this particular length bringing them into the range of typical stimuli used with humans. The strategy was to be able to test for the capability of showing not only latency differences between repeating and random sequences overall, but at every position within the sequence.

Finally, our procedure was designed to allow monitoring of potential learning effects on a daily basis, rather than after a fixed number of acquisition trials or sessions, as has been typical in earlier studies (e.g., Froehlich et al., 2004; Locurto et al., 2010). Instead, our goal was to terminate a given condition when a subject had demonstrably learned the sequence of interest, or alternatively, proved unlikely to learn it. Furthermore, although many SRT studies rely on a blocked design presenting one or more alternating sets of repeating versus randomized trials, in the present procedure, we interspersed these sequence-types, as also has been done in a number of human studies (see Kaufman, DeYoung, Gray, Jimenez, Brown, \& Mackintosh, 2010). This approach was advantageous in that sessions were not of fixed length.
This approach can also be important when subjects are tested repeatedly in order to obviate any effects that trialto-trial predictability might have on performance with repeating versus randomized sequences.

Overview of the procedure To reduce and standardize the response, thereby minimizing inherent latency variability and maximizing the power to detect even small learning effects, the task described presently combined a symmetrical layout of item locations with simple joystick deflection. By using a symmetrical layout, the motor component of responding can be decreased to directional movement alone - in other words, eliminating any differential distance component. By using a joystick, the subject's motion is also reduced and largely, if not wholly, standardized. Finally, using deflection eliminates the need for dexterity, course correction, and sustained attention inherent to full-fledged cursor movement (e.g., Turner et al., 2005).

This symmetrical-response serial reaction time (SR-SRT) task is based on displaying a solid, colored circle in up to eight locations on a computer screen located outside the test chamber. Possible locations are arranged in a circular pattern, and subjects respond to the appearance of a stimulus anywhere on that imaginary perimeter by deflecting a joystick in that direction (see Fig. 1). This approach was implemented with rhesus monkeys and will be described in the form of a method section followed by several testing proto-
Fig. 1 a Schematic illustrations of the cursor and start box seen by the monkey at the beginning of each trial. b The layout of the circle locations on the screen (lighter colored circles mark the additional four locations used in preliminary training). c A hypothetical 1-4-2-3 sequence and corresponding joystick movements tracking each circle as it appears (a)

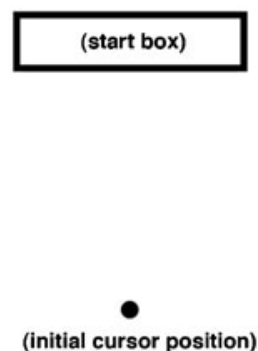

(c)

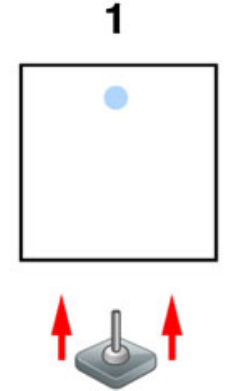

(b)

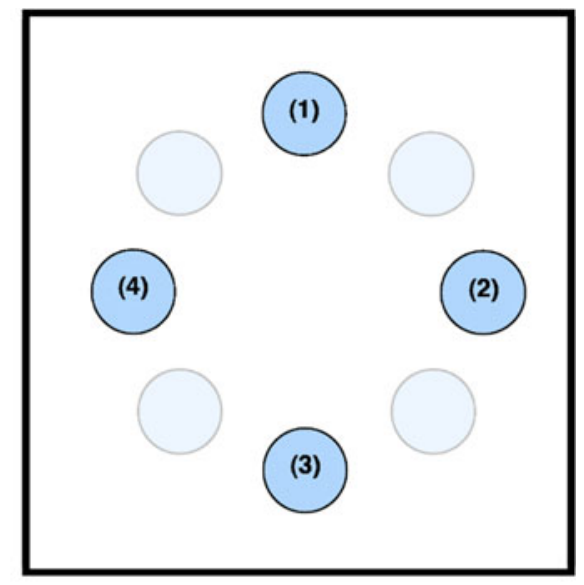

4

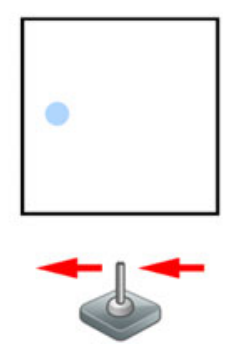

2

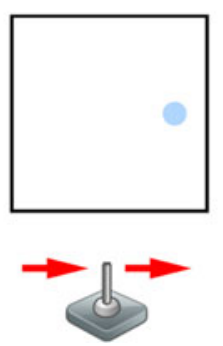

3

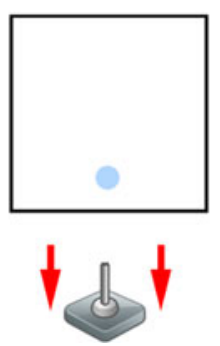


cols. In preliminary training, four monkeys learned to track items presented in all possible locations, in randomized order, and with a sequence length of up to eight items (L8). In two subsequent experiments, the number of locations was reduced to four, and in the second experiment, sequence lengths were initially shorter but were then worked up to eight. In Experiment 1, we tested whether the monkeys would develop significantly faster responses to a repeating sequence than to interspersed, randomized sequences. The three animals that succeeded moved to Experiment 2, which asked whether these animals would also eventually show faster responses to each of the individual positions within the repeating sequence relative to randomized versions.

\section{General method}

\section{Subjects}

Subjects were four male rhesus monkeys that were individually housed at the Language Research Center (LRC) at Georgia State University. At the beginning of testing, one monkey was a juvenile (Obi), and three were adults (Luke, Lou, Hank). All were experienced in using a joystick to respond to stimuli presented on a computer monitor, but none had performed the present task. The monkeys were neither food- nor waterdeprived, in accordance with LRC institutional policy. They had continuous access to several computerized, experimental tasks daily that were performed for food reward. Each task was presented separately through the day for blocks of time ranging from 4 to $24 \mathrm{hr}$. The animals participated in our SR-SRT task approximately three sessions per week. They completed varying numbers of trials in each session, depending on motivation level and session length.

\section{Apparatus}

Subjects were tested individually in their home cages using the LRC Computerized Test System (Richardson, Washburn, Hopkins, Savage-Rumbaugh, \& Rumbaugh, 1990; Washburn \& Rumbaugh, 1992), which included a desktop PC and a 17in. $(43-\mathrm{cm})$ super VGA monitor positioned $24 \mathrm{~cm}$ from the cage behind a transparent Lexan panel. Each monkey had access to its own testing station and controlled a cursor on the monitor using a vertically mounted joystick protruding into the cage. The joystick was approximately centered on the screen horizontally, near the bottom of the monitor. Customwritten Visual Basic 6.0 (Microsoft Corp., Redmond, WA) software controlled trial presentation and data collection, and task performance was tallied using custom scripts. Statistical analyses were conducted with VassarStats (faculty.vassar.edu/ lowry/VassarStats.html) and GraphPad QuickCalcs (www. graphpad.com/quickcalcs).
Design and procedure

Trial contingencies To begin a trial, a monkey used its joystick to move a visible cursor into contact with a start box icon in the middle of the screen (see Fig. 1a). The start box disappeared, and a 1.5-in. blue circle appeared in one of multiple possible positions. These locations were arranged in a circular pattern that was $14.2 \mathrm{~cm}$ in diameter (see Fig. 1b). The monkey moved the joystick in the direction of the stimulus, with any detectable deflection registered as a response (see Fig. 1c). Deflection had to fall within 22.5 degrees of the directional heading to the circle, which caused it to disappear. Both the virtual cursor and the starting point for the next joystick deflection then reset to the center. During a sequence, each correct deflection caused a new stimulus to appear in a different location after a 1-s inter-stimulus interval, without reward.

Completing the sequence triggered delivery of a $97-\mathrm{mg}$ food pellet, a brief, upswept crescendo sound, and a 1-s intertrial interval. The monkey retrieved the reward from a food cup just outside its cage, typically using its left hand, while leaving its right hand on the joystick. For motivational reasons, one monkey (Obi) was rewarded with two pellets throughout. Inaccurate deflection, or failure to respond to a given stimulus within $3 \mathrm{~s}$, triggered a 1-s time-out, and the screen went blank. Data analysis included only sessions in which a subject initiated at least 100 trials and completed at least $80 \%$ of the total trials it started. A basic performance criterion for moving ahead was to meet these criteria in at least three of four consecutive sessions. Approximately $7 \%$ to $10 \%$ of sessions were discarded over the course of the experiments.

Sequence presentations and data analysis For clarity, we will use location to refer to place on the monitor screen, whereas position will refer to place within the stimulus sequence. Although eight locations were available, the experiments used a subset of four, numbered clockwise from the top of the display (see Fig. 1b). Testing sequences routinely included up to eight positions, with repeating sequences consisting of up to eight items appearing in a predetermined order. Randomized sequences included up to eight pseudorandomly determined items, subject to the constraint that the same location could not immediately repeat.

Data collected for each stimulus presentation included sequence type, screen location, sequence position, and corresponding response latency in milliseconds. Trial-bytrial data included whether the sequence was completed and rewarded versus not completed and ending in timeout. Session tallies included trials completed by sequence type and median latency means on completed trials by location and position. Performance on incomplete sequences was not analyzed. Overall and positional latency performance 
with repeating versus randomized sequences were compared session-by-session using one-tailed, unpaired $t$ tests.

Preliminary training The four monkeys first practiced with randomized sequence lengths of one (L1) to four (L4) using all eight screen locations. Each animal was moved ahead to the next training sequence length as it learned to complete each regimen's sequences quickly and reliably. Animals were next trained with L4, L6, and L8 randomized sequences, and were required to meet the performance criteria described earlier in each case. All monkeys completed this phase, requiring a mean of 19.5 test sessions $(S D=4.9)$.

\section{Experiment 1}

Method

Sessions included both repeating and randomized trials. Repeating trials occurred with $90 \%$ probability and presented the
L8 sequence 42313241. This particular sequence was chosen because each item $(1,2,3,4)$ occurs twice, and each transition in the sequence (e.g., 4-2, 2-3, and so on) occurs just once. The remaining $10 \%$ of trials were randomized and randomly interspersed with repeating-sequence trials. A monkey completed this experiment when showing statistically faster overall mean latency on the repeating than on the randomized sequence types for three of four consecutive sessions.

Results and discussion

Illustrative results are shown in Fig. 2. Two monkeys (Obi and Luke) met criterion after 13 and 6 sessions, respectively ( $p<.01$ for each criterion session, for each monkey). Lou progressed more slowly, requiring 65 sessions to complete the experiment, whereas Hank never reached criterion and was dropped from the study. However, reaching criterion performance overall did not imply showing faster RTs at all positions within the sequence. Obi and Luke were therefore continued on the testing regimen for an additional three months as Lou continued training. Comparing mean

\section{Repeating $\diamond \quad$ Randomized $\square$}
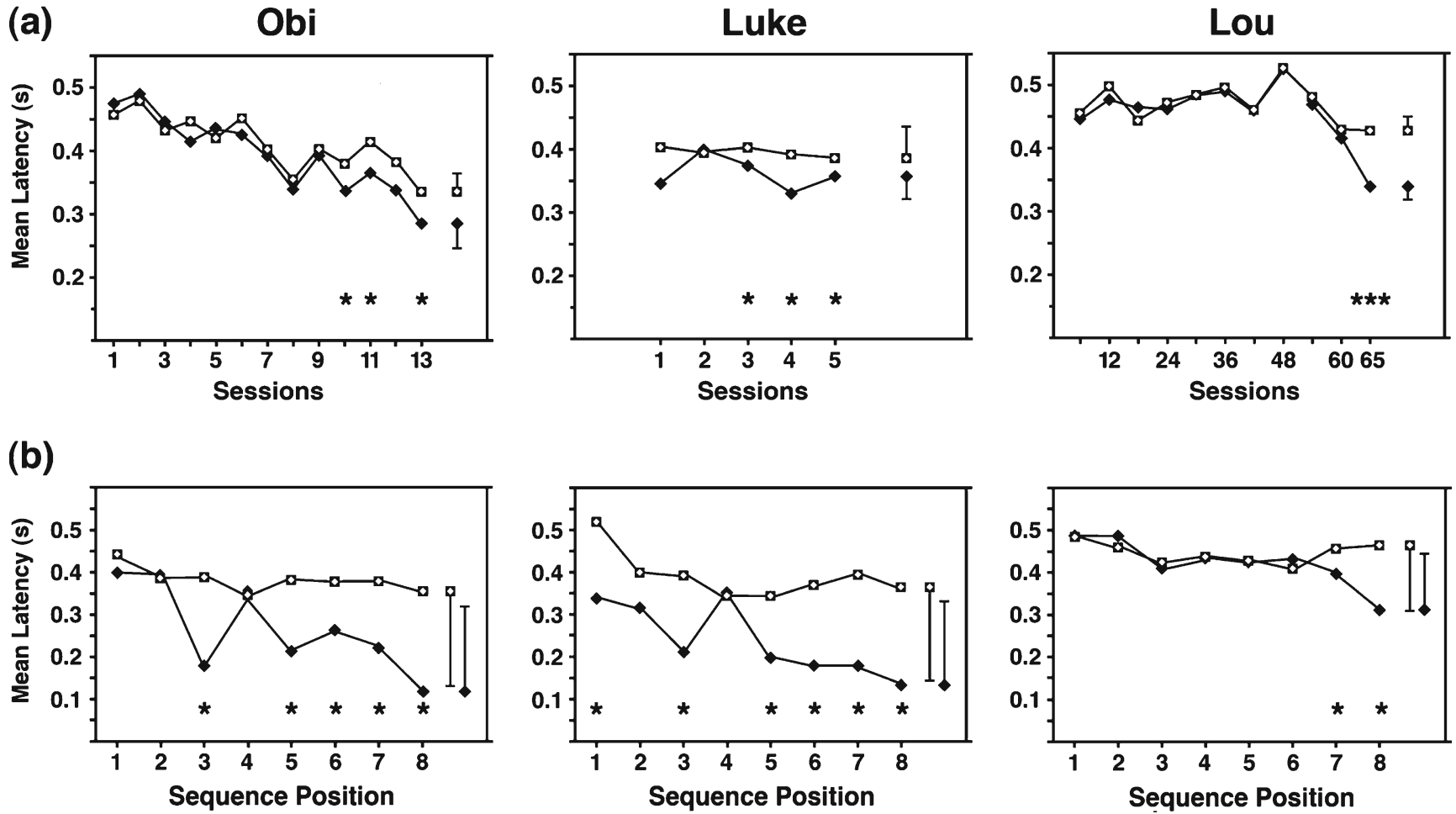

Fig. 2 Performances shown are for the three monkeys that were successful learning the eight-item repeating sequence in Experiment 1. a Individual session performance shown for Obi and Luke reveals rapid progress across individual sessions, whereas Lou's data are shown for blocks of 10 sessions because of his slower progress. Asterisks mark the individual three of four sessions in which overall mean latencies were lower on repeating versus randomized trials, noting that Lou's were all in the last block of his sessions (no distinctions are made based on level of significance). b Position-by-position performance over the last 3 days of testing revealed a number of instances of statistically different reaction times for repeating versus randomized sequences (marked by asterisks), but with uneven outcomes across animals. For clarity, only a single pair of errors bars is shown in each figure, representing the mean standard deviations across all sessions or positions 
latencies by position within completed repeating versus randomized trials over the final three days of this testing revealed that Obi was statistically faster at positions $3,5,6$, 7 , and 8 (all $p \mathrm{~s}<.05$ ), whereas Luke was statistically faster at positions $1,3,5,6,7$, and 8 (all $p$ s $<.05$ ). However, neither monkey showed signs of further improvement. Upon reaching criterion, Lou's performance was less differentiated, showing statistical differences between sequence types at positions 7 and 8 only ( $p$ s $<.01)$.

Results thus showed that three of four monkeys learned to respond faster overall to a repeating sequence of four locations and eight items than to corresponding, randomized versions. Two of the animals progressed steadily, whereas progress for the third was slow and less certain. Furthermore, position-by-position effects indicated that none of the three subjects had learned the sequence in its entirety. All three animals did show differences at the last two positions, consistent with their proximity to reinforcement (Locurto et al., 2009, 2010). In addition, early positions can involve initial uncertainty as to whether a given trial involves a repeating or randomized sequence. Continued training on the same regimen improved position-based performance for the two best-performing monkeys, but they still did not reach fully consistent performance. Experiment 2 was therefore designed to test whether the animals could demonstrate faster responses at every position.

\section{Experiment 2}

As was noted earlier, a potential advantage of responding via joystick deflections with a symmetrical stimulus layout is the potential power to reveal even modest learning effects. In Experiment 2, we capitalized on this sensitivity to test whether possibly subtle, yet convincing "latency separation" could be achieved through restarting training at a shorter sequence length. Sequences were then gradually extended, with the goal of requiring latency separation at every position in order to achieve criterion performance.

\section{Method}

Subjects were monkeys Obi, Luke, and Lou. Testing was conducted as before, except that each monkey progressed through L5, L6, L7, and L8 sequences as criterion performances were met. As a standardized starting point, the animals began by practicing with 3,2,4,1-the last four locations from their familiar fixed sequence. Obi and Luke were then assigned unique L5 sequences, in order to decrease dependence on previous learning and thereby better test the efficacy of the shaping process. Because of his slower, previous performance, however, Luke's L5 sequence also began with $3,2,4,1$. With additional positions added one-by-one, full-length sequences eventually became $4,2,3,1,3,2,1,4$ for Obi; 4,3,2,1,2,4,1,3 for Luke; and $3,2,4,1,2,3,1,4$ for Lou (see also Table 1).

Results and discussion

Although the time needed for each monkey to learn the fixed sequence in Experiment 1 varied, all three animals performed well during the first day of preliminary practice with L4. Here, Obi was statistically faster at positions 3 and $4(p s<.0001)$, Luke was statistically faster at all four sequence positions (all $p \mathrm{~s}<.0001$ ), and Lou was statistically faster at positions $2(p<.05)$ and $4(p<.0001)$. Although Luke and Obi then reached criterion performance on three of four consecutive days and began Experiment 2 after seven sessions, Lou required 72 sessions. Subsequent results for sessions to criterion at L5 to L8 are shown in Table 1. Both Obi and Luke moved quickly on each sequence, although Obi struggled with position 2 on some, even after extended practice. In two instances-L7 and L8-he was therefore considered to have met criterion when achieving statistical separation at all other positions. Lou required more extensive training throughout, and also struggled at position 2 in one instance.

Overall, the animals did achieve statistical separation between latencies on repeating versus randomized trials over three of four sessions at each position at L8, except for one troublesome position for Obi. Continuing on that regimen, however, ongoing performance was not found to be reliable across the board. As illustrated in Fig. 3, achieving clear latency separation at every position required averaging over multiple sessions. As shown, 10 sessions were sufficient for Luke, whereas additional sessions were

Table 1 Experiment 2: sequence length information for each monkey

\begin{tabular}{llll}
\hline Subject & Sequence length & Sequence & Sessions to criterion \\
\hline Obi & 5 & 42,313 & 12 \\
& 6 & 423,132 & 4 \\
& 7 & $4,231,321$ & $3^{*}$ \\
& 8 & $42,313,214$ & $5^{*}$ \\
Luke & 5 & 43,212 & 8 \\
& 6 & 432,124 & 3 \\
& 7 & $4,321,241$ & 3 \\
& 8 & $43,212,413$ & 6 \\
Lou & 5 & 32,412 & 26 \\
& 6 & 324,123 & $36^{*}$ \\
& 7 & $3,241,231$ & 34 \\
& 8 & $32,412,314$ & 31 \\
\hline
\end{tabular}

* No significant difference between conditions at position 2 
needed to demonstrate statistical separation at all positions for the other two. The experiment thus does demonstrate reliable differentiation of RTs in repeating versus randomized sequence positions for rhesus monkeys, with the caveat that statistical separation is most robust when data are averaged over blocks of sessions and for individuals working well at the task from the beginning.

\section{General discussion}

Taken together, results first demonstrated that all four rhesus monkeys tested in a joystick-based SRT procedure could reliably track a randomized sequence of items. Three of four subjects then showed significantly faster latencies on a variety of repeating-sequence lengths when compared to corresponding randomized versions. In Experiment 1, small but reliable overall effects were quickly evident at L8 for two subjects, whereas the third progressed much more slowly. However, showing faster performance overall did not imply latency separation at each individual sequence position. The latter proved challenging for all three monkeys, although each ultimately succeeded in Experiment 2. Here, the strategy of gradually increasing sequence lengths was likely a key factor.

\section{Individual differences and training time}

Several notable individual differences emerged in task performance. One was the larger number of sessions required for Lou to meet criterion performances in both experiments. Furthermore, there were some instances in which a particular position proved challenging for a given monkey in Experiment 2. Position 2 was the most problematic, which may have been because of the inherent difficulty of distinguishing repeating versus randomized trials at this early stage. Learning may also have been slowed overall by the increasing probability of overlap between the positional transitions of repeating versus randomized versions with longer sequences. Proximity to reward would be expected to facilitate both learning and motivation toward the end of any given sequence (Locurto et al., 2009, 2010), with observed performance usually becoming faster at the final positions. However, the monkeys generally overcame their position-related difficulties as they practiced at each length.

Individual differences are to be expected when working with nonhuman primates (e.g., Locurto, 2007). Although it is not clear whether individual subjects were dropped from previous primate SRT studies as well, the animal eliminated from the present Experiment 1 was likely unmotivated rather than unable to track a repeating sequence. Furthermore, performance variability among the three successful monkeys may have been exacerbated by the fact that these subjects worked in their home cages without specific food restriction. This interpretation is corroborated by observed variation in the mean number of trials completed per session. In Experiment 1, those means were 226 for Obi, 615 for Luke, and 234 for Lou. On L8 testing in Experiment 2, these animals completed on average 335, 851, and 577 trials, respectively. Overall, all three subjects were probably working

\section{Repeating $\diamond \quad$ Randomized $\square$}

Obi
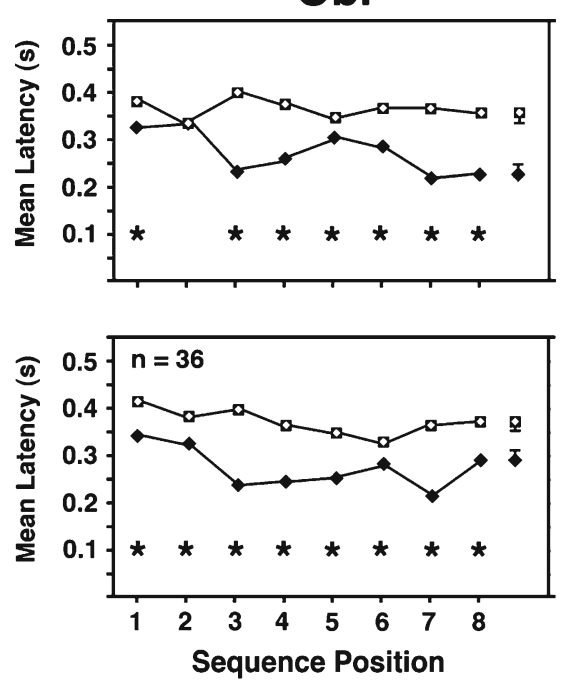

Luke
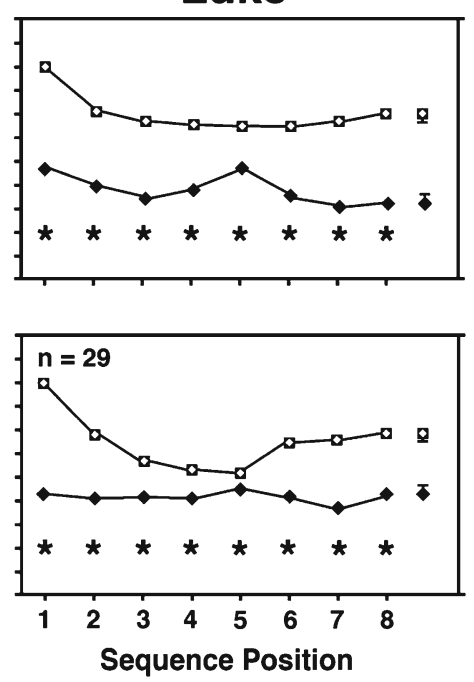

Lou
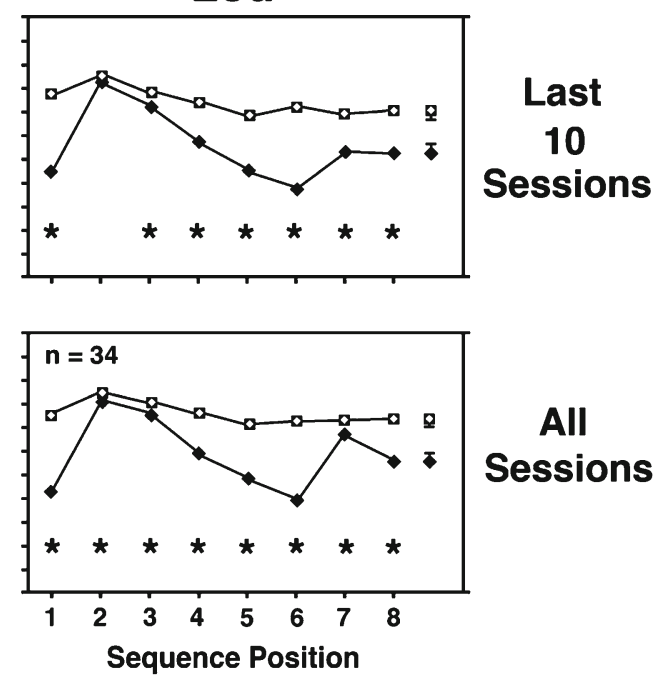

Fig. 3 Position-by-position performance in Experiment 2 at the sequence length of eight for the three monkeys. Overall outcomes are shown for the last 10 sessions of testing and for all test sessions (number of sessions noted for each animal). In both instances, sessions included were those when the animal met criterion performance. For clarity, only a single pair of errors bars are shown in each figure, representing the mean standard errors across all positions 
at a slower pace than they would have if a stricter food regimen had been in effect, and if they hadn't been subject to colony-room distractions.

Nonetheless, each animal completed the entire sequence on the large majority of trials. Although the data collected did not distinguish between time-outs associated with excessively slow responding and possible errors in joystick deflection, neither was common. The three animals consistently showed completion rates of over $85 \%$, and were more often above $90 \%$. Given the simplicity of the task, the majority of incomplete trials likely reflected motivational lapses and distractions rather than deflection errors. However, future implementations of the procedure can benefit from distinguishing between time-outs occurring because of slow responding versus errors in joystick movement.

Training was also likely affected by overlap in the transitions making up the repeating and random sequences used. As was noted earlier, previous SRT studies with nonhumans have constrained the form of randomized sequences in order to facilitate comparison to repeating versions (e.g., Christie \& Dalrymple-Alford, 2004; Locurto et al., 2009, 2010). The strategy adopted presently was to allow comparison sequences to be as random as possible, subject only to the constraint that a given item could not repeat. On the one hand, then, the probability of exact duplication between randomized and repeating sequences was quite small (1/108 for L4, 1/324 for L5, 1/972 for L6, 1/2916 for L7, and 1/8748 for L8). On the other hand, there was a substantial probability of overlap in one or more constituent transitions, at least if disregarding sequence position (approximately $52 \%$ for L4, approximately $77 \%$ for L5, and over $95 \%$ for L8). Overlap between repeating and randomized sequences may thus have slowed the learning process, potentially also exacerbating individual differences in acquisition. Taking these factors into account, we suspect that the time and effort involved in bringing subjects to the various performance levels demonstrated here were notably greater than were strictly required - depending on the design and experimental question involved in a given implementation.

\section{Conclusions}

Our primary motivation for developing the SR-SRT task was to then be able to investigate what rhesus monkeys can learn about probabilistically structured sequences-both overall and item-by-item (Heimbauer et al., 2011). In that work, the same subjects tested presently had been trained on sequences generated by underlying grammar-like rules and were tested for generalization to novel, rule-based versus randomized instances. Using eight-item sequences was critical, since shorter sequences provide less opportunity to compile large, independent sets of training and testing exemplars. The subsequent experiments also benefited from using a standardized response form, avoiding the need to control for location-based latency variability. In the present work, standardization was arguably evident in finding that latencies were uniformly fast, both overall and across sequence positions. That speed of response furthermore implies that the monkeys were, as hoped, making easy, small movements that could be performed many times in each session over an extended period of training and testing. Here, even the least hardworking animals were routinely performing more than 3,000 individual joystick deflections per session.

The present procedure was also successful in bringing subjects up to sequence lengths falling within the range of those used with humans. In Experiment 1, for example, two subjects showed immediate and rapid progress toward faster responding on an eight-item sequence. All three monkeys then worked well when moved back to shorter repeating sequences in Experiment 2, suggesting specific learning of at least a subset of individual positions in the longer versions. Although the robust separation of response latencies on repeating versus randomized L8 trials being sought in this second study required much more extensive training, it was achievable. Here, the goal was to demonstrate that, like humans, the subjects were learning about every element within the sequence. That was not clearly the case in Experiment 1; however, all three subjects showed consistent overall interference effects that could nonetheless have reflected learning about a subset of sequence items and transitions.

The same caveat applies to two previous studies with nonhumans that have shown interference effects at lengths of eight or more items. Although Turner et al. (2005) tested monkeys with 10-item sequences, these sequences were composed of repeating triplets. Effective sequence length was therefore significantly less than the number of individual items involved. Working with rats, Christie and Dalrymple-Alford (2004) used sequences that did not repeat, and they demonstrated interference effects at lengths of both eight and 12 items. However, these were overall differences shown over blocks of 30 sequences each, and the subjects were tested for only one session. It is therefore not known how much the rats learned about each sequence, and the results could reflect learning about just a few items or transitions. It is therefore quite unlikely that the rats were learning every element in the sequences.

Overall, we suggest that the SR-SRT, joystick-based procedure described presently can be useful across a variety of circumstances. The procedure is easy to implement and lends itself both to shorter and longer sequences, and to rapid as well as extended testing procedures. A drawback in the present approach was the extended training time involved. However, that training was designed to meet the rather draconian requirements of our particular research 
program and is not inherent to the method itself. In fact, we suggest that the overall approach is quite flexible, need not require any more training time than other procedures used with nonhumans, and can provide an additional tool for investigating an important topic in nonhuman cognition.

Author Note This research was supported in part by National Institutes of Health Grants HD-38051 and HD-060563, a Research on the Challenges of Acquiring Language \& Literacy (RCALL) seed grant, and the College of Arts and Sciences at Georgia State University. L. H. was an RCALL Fellow and Duane M. Rumbaugh Fellow during completion of this work.

\section{References}

Christie, M. A., \& Dalrymple-Alford, J. C. (2004). A new rat model of the human serial reaction time task: Contrasting effects of caudate and hippocampal lesions. Journal of Neuroscience, 24, 1034-1039.

Christie, M. A., \& Hersch, S. M. (2004). Demonstration of nondeclarative sequence learning in mice: Development of an animal ana$\log$ of the human serial reaction time task. Learning \& Memory, 11, 720-723.

Cleeremans, A., \& McClelland, J. L. (1991). Learning the structure of event sequences. Journal of Experimental Psychology: General, 120, 235-253.

Domenger, D., \& Schwarting, R. K. (2005). Sequential behavior in the rat: A new model using food-reinforced instrumental behavior. Behavioral Brain Research, 160, 197-207.

Domenger, D., \& Schwarting, R. K. (2007). Sequential behavior in the rat: Role of skill and attention. Experimental Brain Research, 182, 223-231.

Froehlich, A. L., Herbranson, W. T., Loper, J. D., Wood, D. M., \& Shimp, C. P. (2004). Anticipating by pigeons depends on local statistical information in a serial response time task. Journal of Experimental Psychology: General, 133, 31-45.

Gabrieli, J. D. E. (1998). Cognitive neuroscience of human memory. Annual Review of Psychology, 49, 87-115.

Heimbauer, L. A., Conway, C. M., Christiansen, M. H., Beran, M. J., \& Owren, M. J. (2011). [Grammar-rule learning and generalization by rhesus macaques]. Unpublished data.

Hunt, R., \& Aslin, R. N. (2001). Statistical learning in a serial reaction time task: Access to separable statistical cues by individual learners Journal of Experimental Psychology: General, 130, $658-680$

Jamieson, R. K., \& Mewhort, D. J. K. (2005). The influence of grammatical and local and organizational redundancy on implicit learning: An analysis using information theory. Journal of Experimental Psychology: Learning, Memory and Cognition, 31, 9-23.
Kaufman, S. B., DeYoung, C. G., Gray, J. R., Jimenez, L., Brown, L., \& Mackintosh, N. (2010). Implicit learning as an ability. Cognition, 116, 321-340.

King, W. J. (1961). Continuous compensatory tracking by a cebus monkey. Science, 134, 947-948.

Lee, D. (2003). Coherent oscillations in neuronal activity of the supplementary motor area during a visuomotor task. Journal of Neuroscience, 23, 6798-6809.

Lee, D., \& Quessy, S. (2003). Activity in the supplementary motor area related to learning and performance during a sequential visuomotor task. Journal of Neurophysiology, 89, 1039-1056.

Locurto, C. (2007). Individual differences and animal personality. Comparative Cognition \& Behavior Reviews, 2, 67-78.

Locurto, C., Gagne, M., \& Levesque, K. (2009). Implicit chaining in cotton-top tamarins (Saguinus oedipus). Journal of Experimental Psychology: Behavior Processes, 35, 116-122.

Locurto, C., Gagne, M., \& Nutile, K. (2010). Characteristics of implicit chaining in cotton-top tamarins (Saguinus oedipus). Animal Cognition, 13, 617-629.

Nissen, M. J., \& Bullemer, P. (1987). Attentional requirements of learning: Evidence from performance measures. Cognitive Psychology, 19, 1-32.

Procyk, E., Dominey, P. F., Amiez, C., \& Joseph, J.-P. (2000). The effects of sequence structure and reward schedule on serial reaction time learning in the monkey. Cognitive Brain Research, 9, 239-248.

Richardson, W. K., Washburn, D. A., Hopkins, W. D., SavageRumbaugh, E. S., \& Rumbaugh, D. M. (1990). The NASA/LRC computerized test system. Behavior Research Methods, 22, 127131.

Robertson, E. M. (2007). The serial reaction time task: Implicit motor skill learning? Journal of Neuroscience, 27, 10073-10075.

Thomas, K. M., \& Nelson, C. A. (2001). Serial reaction time learning in preschool- and school-age children. Journal of Experimental Child Psychology, 79, 364-387.

Turner, R. S., McCairn, K., Simmons, D., \& Bar-Gad, I. (2005). Sequential motor behavior and the basal ganglia. In J. P. Bolam, C. A. Ingham, \& P. J. Magill (Eds.), The basal ganglia VIII: Advances in behavioral biology (Vol. 56, pp. 563-574). Singapore: Springer.

Washburn, D. A., Beran, M. J., Evans, T., Hoffman, M., \& Flemming, T. (in press). Technological innovations in comparative psychology: From the problem box to the "Rumbaughx." In L. L'Abate \& D. Kaiser (Eds.). Handbook of technology in psychology, psychiatry, and neurology. Hauppauge, NY: Nova Science Publishers.

Washburn, D. A., \& Rumbaugh, D. M. (1992). Testing primates with joystick-based automated apparatus: Lessons from the Language Research Center's computerized test system. Behavior Research Methods, 24, 157-164.

Willingham, D. B. (1999). Implicit motor sequence learning is not purely perceptual. Memory \& Cognition, 27, 561-572.

Willingham, D. B., \& Goedert-Eschmann, K. (1999). The relation between implicit and explicit learning: Evidence for parallel development. Psychological Science, 10, 531-534. 